The 13th European Nutrition Conference, FENS 2019, was held at the Dublin Convention Centre, 15-18 October 2019

\title{
Parental perception of physical activity among school children aged 7 years in Montenegro
}

\author{
Zorica Đorđević, Enisa Kujundžić and Borko Bajić \\ Institute of Public Health, Podgorica, Montenegro
}

\section{Abstract}

Introduction: Globalisation and modern lifestyles are contributing to different public health problems, especially in early age.

Objectives: To present the result of family questionnaires regarding the parental perception of physical activity in children and to discuss the importance of findings compared to overweight and obesity rates in this population group.

Methodology: In Montenegro Childhood obesity surveillance initiative (COSI) including 7 years old school children was conducted for the first time in 2016 in accordance with WHO protocol. In total 3581 children were examined and 3162 family questionnaires collected. We analyzed a part of the family questionnaires dealing with children free time in correlation with the results of anthropometric measurements.

Results: In working days, according to the parents' claims, around $73 \%$ of children spend more than an hour playing outside, while at weekends that number is around $95 \%$. Spending free time in front of the screen longer than 4 hours is bigger at weekends comparing to working days 14\%:4\%. The results of anthropometric measurement show that around 1/5 of boys and girls are overweighed and around over $1 / 5$ of boys and $1 / 10$ are obese.

Conclusion: Parental perception of physical activity level among children is one of the key factors that could influence children growth and development into healthy, resilient adults. Public health approach in strengthening this perception is rising awareness of knowledge, attitudes and behavior of parents regarding the connection between physical activity, free time and overweight and obesity rates in their children.

\section{Conflict of Interest}

No 